\title{
Manejo de Diabrotica speciosa com atrativos naturais em horta orgânica ${ }^{1}$
}

\author{
João José Stüpp²; Mari Inês C Boff ${ }^{3}$; Paulo Antonio de S Gonçalves ${ }^{4}$ \\ ${ }^{2}$ Escola Agrotécnica Federal de Rio do Sul, C. Postal 441, 89160-000 Rio do Sul-SC; ${ }^{3}$ UDESC-CAV, C. Postal 281, 88520-000 Lages- \\ SC; ${ }^{4}$ Epagri-Est. Exp. de Ituporanga, C. Postal 121, 88400-000 Ituporanga-SC; E-mail: a8jjs@cav.udesc.br; a2micb@cav.udesc.br
}

\section{RESUMO}

O controle de insetos associados ao cultivo de hortaliças é predominantemente realizado através de pesticidas sintéticos. Entretanto, os riscos do uso indiscriminado dos pesticidas sobre a saúde humana e o meio ambiente deve ser considerado. O objetivo deste trabalho foi estudar a eficácia de atrativos naturais no controle da vaquinha Diabrotica speciosa (Germar) (Coleoptera: Chrysomelidae), uma praga de importância econômica na América Latina. Os seguintes tratamentos foram testados: raiz de taiuiá (Cayaponia sp), frutos verdes de porongo/cuia (Lagenaria sp), extrato de folhas de couve-chinesa (Brassica pekinensis), fermentado de frutas, vinagre tinto e água como testemunha. $\mathrm{O}$ delineamento foi inteiramente ao acaso, com seis repetições. Os preparados foram acondicionados em armadilhas com garrafas PET de $2.000 \mathrm{ml}$, com oito aberturas laterais e distribuídas aleatoriamente na área experimental. Frutos verdes de porongo apresentaram captura de adultos de D. speciosa 5,4 vezes maior que raízes de taiuiá e as raízes de taiuiá capturaram 19,7 vezes mais adultos do que a testemunha. Ambas as iscas foram acondicionadas na forma sólida, suspensas internamente na garrafa PET. A menor atratividade foi apresentada pelo extrato aquoso de couve-chinesa, que não diferiu da testemunha. Independentemente do atrativo utilizado, o pico populacional da $D$. speciosa ocorreu nos meses de fevereiro e março.

Palavras-chave: Lagenaria sp., Cayaponia sp., atrativos naturais, armadilhas.

\section{ABSTRACT}

Natural attractios for management of Diabrotica speciosa in a organic home garden

The control of insects associated with home garden vegetable crops is usually done by sintetic pesticides. However, the risks of the indiscriminate use of pesticides on human health and the environment need to be considered. The objective of this work was to study the attractive efficacy of natural substances on D. speciosa, a very important pest in Latin America. The following treatments were tested: roots of Cayaponia sp., green fruits of Lagenaria sp., water extract of Brassica pekinensis, extract of fermented fruits, red vinegar and water as a control. The experimental design was randomized blocks with six replications. All the tested substances were put into PET plastic bottles of $2 \mathrm{~L}$ with eight lateral holes and distributed at random in the experimental area. Green fruits of Lagenaria sp. captured the larger number of D. speciosa, 5.4 times more than roots of Cayaponia sp. that captured 19.7 times more than the control tratament. The water extract of Brassica pekinensis had no effect on D. speciosa attraction, and did not differ from the control treatment in the number of captured insects. Independently of the treatment, the population peak of D. speciosa occurred in February and March.

Keywords: Lagenaria sp., Cayaponia sp., vegetables, natural attractive, traps.

(Recebido para publicação em 20 de março de 2006;

aceito em 6 de dezembro de 2006)

\begin{abstract}
$\mathrm{A}^{\mathrm{s}}$ s hortaliças constituem o principal grupo de alimentos com vitaminas e sais minerais, fundamentais para a saúde do ser humano em todas suas faixas etárias (Filgueira, 2003). Apesar de a produção brasileira de hortaliças ter sido superior a 14 milhões de toneladas em 2002, o consumo 'per capita' dos brasileiros fica em torno de 52 gramas/dia, enquanto os norte-americanos e alemães chegam a 150 gramas/dia (Embrapa, 2005; Makishima \& Luengo, 2005).
\end{abstract}

Diabrotica speciosa (Germar) (Coleoptera: Chrysomelidae) é praga polífaga de grande importância na América Latina e uma das espécies fitófagas muito freqüentes em cultivos diversos, capaz de causar importantes danos em várias espécies de vegetais (Ventura $e t$ al., 2001). Segundo Milanez (1987), D. speciosa pode provocar perdas totais nas lavouras de feijão, particularmente nos cultivos de safrinha. Conhecida como "vaquinha", "brasileirinho" e "patriota", D. speciosa apresenta ciclo biológico de dois meses e fecundidade superior a 400 ovos por fêmea. As fêmeas depositam seus ovos próximos da base das plantas hospedeiras, cujas raízes são consumidas pelo inseto em sua fase larval (Gassen, 1996), sendo as espécies da família Poaceae as preferidas (Roel \& Zatarin, 2006). As leguminosas, incluindo o feijoeiro, e as cucurbitáceas estão entre as espécies mais atrativas para os adultos de D. speciosa, principalmente na fase de germinação e desenvolvimento inicial. Em infestações severas, essa vaquinha pode causar a morte das plantas (Quintela, 2004). As estratégias de controle da vaquinha, conforme se verifica na bibliografia, são baseadas no emprego de inseticidas químicos (Ventura et al., 2001). Entretanto, Gonçalves \& Boff (2002), afirmam que o uso crescente e abusivo de agrotóxicos tem acarretado sérias consequiências à saúde humana e ao meio ambiente, o que justifica a necessidade urgente de técnicas eficientes e ecologicamente aceitáveis para o manejo populacional desses insetos. Segundo Martinez (2003), o controle químico de D. speciosa é de eficiência insatisfatória devido à contínua reinfestação das áreas, em vista do hábito migratório desta espécie. Assim, métodos de baixo custo e

${ }^{1}$ Parte da dissertação do primeiro autor, apresentada à Universidade do Estado de Santa Catarina, para obtenção do título de Mestre em Produção Vegetal. 
impacto ambiental mínimo, bem como de reduzido risco ao ser humano, estão sendo demandados por agricultores e consumidores. Milanez (1987) testou o efeito atrativo de Cayaponia sp. (taiuiá) sobre $D$. speciosa e concluiu que as raízes dessas plantas apresentam alta atratividade sobre os adultos dessa espécie, podendo constituir-se em prática auxiliar no controle da mesma. Estudos realizados por Arl \& Rinklin (1997) e Claro (2001) também confirmaram as propriedades atrativas da raiz de taiuiá sobre adultos de $D$. speciosa. ArrudaGatti \& Ventura (2003) observaram que a cucurbitacina presente em muitas espécies de plantas da família Cucurbitaceae possui efeitos fagoestimulantes e arrestantes sobre adultos de Diabrotica, com comprovada eficiência. Nishida et al. (1986) e Nishida \& Fukami (1990) determinaram que a cucurbitacina 23,24 dihidrocucurbitacina $\mathrm{D}$ possui alta atratividade para $D$. speciosa $e$ Cerotoma arcuata (Bechyné). Segundo Ventura et al. (2000), há forte evidência de coevolução entre crisomelídeos e cucurbitáceas devido a presença de cucurbitacina em sua composição. O emprego de substâncias atrativas tipo iscas não residuais no manejo de insetos constitui tecnologia coadjuvante apropriada na proteção de cultivos orgânicos. O objetivo deste trabalho foi estudar a eficácia de atrativos naturais de baixo custo e reduzido impacto ambiental sobre a vaquinha $D$. speciosa em uma horta orgânica.

\section{MATERIAL E MÉTODOS}

O trabalho foi realizado na área experimental da Escola Agrotécnica Federal de Rio do Sul-SC, mantida em manejo orgânico. Durante o período experimental, de novembro de 2004 a outubro de 2005, os cultivos predominantes na área eram de hortaliças folhosas, solanáceas, cucurbitáceas e leguminosas. O delineamento experimental foi inteiramente ao acaso, com seis repetições. Os tratamentos constaram de atrativos naturais preparados a partir de: a) raízes de taiuiá (Cayaponia sp.); b) frutos verdes de porongo (Lagenaria sp.); c) extrato aquoso de couve-chinesa (Brassica pekinensis) $10 \% \mathrm{p} / \mathrm{v} ; \mathrm{d}$ ) extrato fermentado da mistura de banana Caturra, laranja pêra, maçã Fuji e mamão formosa, $10 \%$ p/v; e) solução de vinagre tinto a $25 \%$; e f) água como testemunha.

Os atrativos do tipo sólido, como a raiz de taiuiá e o porongo verde, foram preparados na forma de fatias com 15 $\mathrm{cm}$ de comprimento e largura suficiente para permitir sua introdução pelo gargalo da garrafa PET, no interior da qual ficaram suspensas por ganchos de arame flexível, presos à boca da garrafa, de modo a permitir o seu fechamento com a tampa original. $\mathrm{O}$ atrativo de extrato de couve-chinesa foi obtido pela trituração em liquidificador doméstico de 100 gramas de folhas verdes em um litro de água e posterior coagem em peneira de malha fina (49 malhas $\left./ \mathrm{cm}^{2}\right)$. O extrato fermentado de frutas foi obtido a partir da mistura de 200 gramas de banana Caturra, laranja pêra, maçã Fuji e mamão formosa, totalizando 800 gramas. As frutas foram fragmentadas em ralador doméstico manual, misturadas e homogeneizadas em recipiente de vidro fechado, permanecendo 48 horas em temperatura ambiente para fermentação. Em seguida, a mistura foi coada em peneira de malha fina e o extrato obtido foi armazenado em refrigerador, por no máximo um dia, até a aplicação. O extrato fermentado foi utilizado na proporção de $100 \mathrm{ml}$ por litro de solução com água. A solução de vinagre tinto foi obtida pela mistura de $100 \mathrm{ml}$ do vinagre de vinho tinto (Heinig @) em $300 \mathrm{ml}$ de água.

Cada atrativo foi acondicionado em garrafas plásticas tipo "PET” (2000 ml), com quatro janelas longitudinais de $5 \mathrm{x} 1$ $\mathrm{cm}$ posicionadas a $10 \mathrm{~cm}$ da base da garrafa, mais quatro furos de um $\mathrm{cm}$ de diâmetro acima e entre as janelas, constituindo a parcela experimental. As garrafas-armadilhas foram suspensas em suportes de ferro a $20 \mathrm{~cm}$ do solo e distribuídas aleatoriamente na área experimental, num caminhamento em ziguezague. A cada cinco semanas foi realizado um rodízio dos materiais atrativos, de modo que todos estiveram localizados em todos os pontos da área pelo mesmo espaço de tempo.

A retenção dos insetos nas armadilhas com os atrativos sólidos, taiuiá e porongo, foi possível pela adição de 400 $\mathrm{ml}$ de água com três gotas de detergente, na armadilha PET. Nos atrativos líquidos, a retenção foi feita pelo próprio preparado, também na quantidade de $400 \mathrm{ml}$ por armadilha PET.

As avaliações constaram da coleta e contagem semanal, por armadilha, dos insetos adultos de $D$. speciosa e dos demais insetos, separados em ordens taxonômicas. A coleta era feita vertendo-se o líquido do interior da armadilha em uma peneira de malha fina. Em seguida, os insetos retidos eram lavados com água sobre a própria peneira e acondicionados em potes plásticos de $80 \mathrm{ml}$, com tampa, contendo álcool $85 \%$. No laboratório, adultos de D. speciosa eram separados e contados. Os dados foram expressos em números cumulativos mensais e submetidos à análise de variância dos valores transformados para $\log (\mathrm{x}+10)$. As médias foram discriminadas pelo teste de Tukey ao nível de $5 \%$ de probabilidade.

\section{RESULTADOS E DISCUSSÃO}

Todos os materiais testados demonstraram possuir atratividade sobre adultos de $D$. speciosa, exceto o extrato de couve-chinesa que não diferiu estatisticamente da testemunha. As iscas preparadas com frutos verdes de porongo destacaram-se como as mais atrativas à vaquinha tendo capturado 5,4 vezes mais adultos que as raízes de taiuiá. Já as raízes de taiuiá atraíram 19,7 vezes mais adultos de vaquinha do que a testemunha (Tabela 1). Esse resultado corrobora com as observações realizadas por Arl \& Rinklin (1997) e Burg \& Mayer (1999). Segundo Ventura et al. (2005), a atratividade de Lagenaria sp. sobre $D$. speciosa deve-se à presença de cucurbitacina nos frutos, o que foi comprovado por Arruda et al. (2005) em testes com formulações contendo essa substância. A raiz de taiuiá não apresentou o mesmo desempenho descrito por Milanez (1987), embora tenha apresentado desempenho significativamente superior à testemunha (Tabela 1). Gassen (1996) observou que o emprego do taiuiá não foi adotado pelos agricultores, provavelmente em função de reduzidos índices de controle. Este au- 


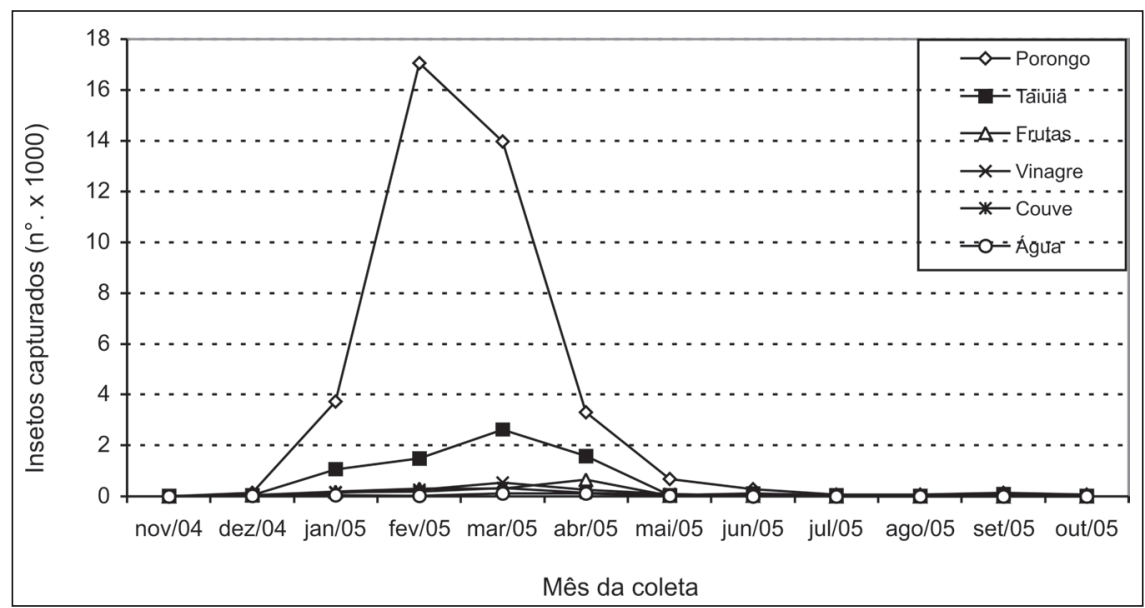

Figura 1. Flutuação populacional de adultos de Diabrotica speciosa capturados em armadilhas contendo diferentes atrativos naturais. Rio do Sul-SC, Escola Agrotécnica Federal de Rio do Sul-SC, 2004/2005.

Tabela 1. Indivíduos adultos de Diabrotica speciosa capturados em avaliações mensais de novembro 2004 a outubro 2005 por atrativos naturais em área de cultivo orgânico de hortaliças. Rio do Sul-SC, Escola Agrotécnica Federal de Rio do Sul-SC, 2004/2005.

\begin{tabular}{lcc}
\hline Substância atrativa & Adultos capturados $\left(\mathrm{n}^{\circ}\right)$ & $\begin{array}{c}\text { Eficiência de atratividade } \\
\text { e captura }\end{array}$ \\
\hline Frutos verdes de porongo & $139,6 \mathrm{a}^{1}$ & 107,3 \\
Raiz de taiuiá & $25,6 \mathrm{~b}$ & 19,7 \\
Extrato fermentado de frutas & $5,6 \mathrm{c}$ & 4,3 \\
Vinagre tinto a 25\% & $4,8 \mathrm{c}$ & 3,7 \\
Extrato aquoso de couve chinesa & $3,6 \mathrm{~cd}$ & 2,7 \\
Água (testemunha) & $1,3 \mathrm{~d}$ & 1.0 \\
\hline CV (\%) & 27,7 \\
${ }^{1}$ Tukey, 5\%: ${ }^{2}$ Cálculo realizado em relação a média de captura do tratamento testemunha.
\end{tabular}

tor observa ainda que estudos recentes evidenciaram que apenas os machos são atraídos pelas raízes de taiuiá, sugerindo uma forma de influência no comportamento sexual desses insetos. Por outro lado, Sanches \& Ishimura (2001) avaliaram que as iscas com sementes de taiuiá são cerca de três vezes mais atrativas que as raízes e cerca de treze vezes mais atrativas que seus caules, recomendando-as como alternativa no controle integrado de D. speciosa.

$\mathrm{O}$ extrato aquoso de couve-chinesa não confirmou sua atratividade natural à $D$. speciosa, conforme apontado por técnicos e produtores que acreditavam ser este um dos extratos mais atrativos, uma vez que as folhas desta hortaliça são altamente infestadas por adultos de vaquinhas. Neste experimento, no entanto, foi o atrativo de menor eficácia, não diferindo estatisticamente da testemunha. $\mathrm{O}$ extrato de salada de frutas no entorno dos cultivos a serem protegidos do ataque da vaquinha, pois os órgãos vegetativos da parte aérea da planta do porongo (ramos e folhas) também exercem forte atração sobre a $D$. speciosa. Esta prática, no entanto, pode ter efeito oposto, atraindo grande número dessas vaquinhas para a área de cultivo, com probabilidade de danos maiores para os cultivos de importância econômica.

Pode-se concluir que frutos verdes de porongo cortados apresentam grande potencial atrativo sobre adultos de $D$. speciosa, constituindo-se em opção viável no manejo integrado deste inseto. Iscas líquidas, à base de fermentado de frutas e vinagre tinto, não apresentam potencial atrativo importante na captura de D. speciosa.

\section{AGRADECIMENTOS}

A Isaías Avi pelo apoio na condução do experimento.

\section{REFERÊNCIAS}

ARL V; RINKLIN H. 1997. Livro Verde 2 Agroecologia. Passo Fundo: Berthier. 68p.

ARRUDA-GATTI IC; VENTURA MU. 2003. Iscas contendo cucurbitacinas para o manejo de Diabrotica spp. Semina: Ciências Agrárias 24: 331-336.

ARRUDA IC; VENTURA MU; SCARMINIO IS. 2005. Feeding and arrestment responses of Diabrotica speciosa to cucurbitacin-content formulations. Pesquisa Agropecuária Brasileira 40: 639-643.

BURG IC; MAYER PH. 1999. Alternativas ecológicas para prevenção e controle de pragas e doenças. 15ª ed. Francisco Beltrão: Grafit. $153 \mathrm{p}$.

CLARO SA. 2001. Referenciais tecnológicos para Agricultura Familiar Ecológica: a experiência da Região Centro-serra do Rio Grande do Sul. Porto Alegre: EMATER/RS-ASCAR. 250p.

EMBRAPA-Empresa Brasileira de Pesquisa Agropecuária, 2004, Linhas de ação: hortaliças. Disponível em http://www.21.sede.embrapa.br/ internet/linhas_de_acao/alimentos/hortaliças/ index html. Acessado em 12 de novembro de 2005.

FILGUEIRA FAR. 2003. Novo manual de olericultura, $2^{\mathrm{a}}$ edição. Viçosa: UFV. 412p.

GASSEN DN. 1996. Manejo de pragas associadas à cultura do milho. Passo Fundo: Aldeia Norte. 134p.

GONÇALVES PAS; BOFF P. 2002. Manejo agroecológico de pragas e doenças: conceitos e definições. Revista Agropecuária Catarinense 15: 51-54. 
MAKISHIMA N; LUENGO R de FA. 2005. Tabela de Composição Nutricional das Hortaliças. Brasília-DF: EMBRAPA.

MARTINEZ SS. 2003. Controle da vaquinha com o inseto triturado. Agroecologia Hoje 4: 22

MILANEZ JM. 1987. Atratividade e Controle de Diabrotica speciosa pelo uso da raiz de tayuyá (Cayaponia spp) na cultura do feijoeiro. Chapecó: $\quad$ EMPASC/CPPP. $\quad 7 \mathrm{p}$. (Mimeografado).

NISHIDA R; FUKAMI H. 1990. Sequestration of distasteful compounds by some pharmacophagous insects. J. Chem. Ecol 16: 151-164.

NISHIDA R, FUKAMI H, TANAKA Y, MAGALHÃES BP, YOKOYAMA M, BLUMENSCHEIN A. 1986. Isolation of feeding stimulants of Brazilian leaf beetles (Diabrotica speciosa and Cerotoma arcuata) from the root of Ceratosanthes hilariana. Agric. Biol. Chem 50: 2831-2836.
QUINTELA ED. 2004. Manejo integrado dos insetos e outros invertebrados pragas do feijoeiro. Informe Agropecuário 25: 113-136.

ROEL AR; ZATARIN M. 2006. Data sheets on quarentine pests, Diabrotica speciosa. EPPO. Disponível em http:/www.eppo.org/ QUARANTINE/insects/Diabroticaspeciosa/ DSDIABSC.pdf, Acessado em 02 setembro 2006.

SANCHES MA; ISHIMURA I. 2001. Atratividade de sementes de taiuiá a Diabrotica speciosa em acelga. Instituto Biológico 68: 97-101.

SUTTER GR; LANCE DR. 1991. New strategies for reducing insecticide use in the corn belt. $\mathrm{p}$. 230-249, In Sustainable agriculture research and education in the field: proceedings. Disponível em http://darwin.nap.edu/ openbook.php. Acessado em 03 setembro 2006.
VENTURA MU; MELLO PP; OLIVEIRA ARM; SIMONELLI F; MARQUES FA; ZARBIN PHG. 2001. Males are attracted by females traps: a new perspective for management of Diabrotica speciosa (Germar) (Coleoptera: Chrysomelidae) using sexual pheromone. Neotropical Entomology 30: 361-364.

VENTURA MU; RESTA CCM; NUNES DH; FUJIMOTO F. 2005. Trap attributes influencing capture of D. speciosa (Coleoptera: Chrysomelidae) on common bean fields. Scientia Agricola 62: 351-356. 